\title{
Research on Noise Reduction Method of Pressure Pulsation Signal of Draft Tube of Hydropower Unit Based on ALIF-SVD
}

\author{
Yan Ren $\mathbb{D}^{1},{ }^{1,2}$ Pan Liu $\mathbb{D}^{1},{ }^{1}$ Leiming Hu, ${ }^{3}$ Jin Huang $\left(\mathbb{D},{ }^{1}\right.$ Ruoyu Qiao, ${ }^{1}$ Hongping Chen, ${ }^{4}$ \\ Xiaokai Li, ${ }^{4}$ and Shaojie Huang ${ }^{5}$ \\ ${ }^{1}$ School of Electric Power, North China University of Water Resources and Electric Power, Zhengzhou 450045, China \\ ${ }^{2}$ Hunan Provincial Key Laboratory of Renewable Energy Power Technology (Changsha University of Science and Technology), \\ Changsha 410114, China \\ ${ }^{3}$ Jiangxi Hongping Pumped Storage Co., Ltd., Yichun 330600, China \\ ${ }^{4}$ State Grid Hunan Electric Power Co., Ltd., Changsha 410004, China \\ ${ }^{5}$ Henan Nuclear Industry Radionuclide Testing Center, Zhengzhou 450046, China
}

Correspondence should be addressed to Pan Liu; 773793250@qq.com

Received 27 January 2021; Revised 5 March 2021; Accepted 13 March 2021; Published 31 March 2021

Academic Editor: Ling Zhou

Copyright ( $\odot 2021$ Yan Ren et al. This is an open access article distributed under the Creative Commons Attribution License, which permits unrestricted use, distribution, and reproduction in any medium, provided the original work is properly cited.

\begin{abstract}
Aiming at the problem that it is difficult to extract the characteristics of the draft tube pressure fluctuation signal under the background of strong noise, this study proposes a dual noise reduction method based on adaptive local iterative filtering (ALIF) and singular value decomposition (SVD). First, perform ALIF decomposition of the signal to be decomposed to obtain a series of IMF components, calculate the sample entropy of each component, select some IMF components to reconstruct according to the set sample entropy threshold, and then perform SVD decomposition on the reconstructed signal, and according to the location of the singular value difference spectrum mutation point, the appropriate number of reconstructions is selected for reconstruction, so as to achieve the double noise reduction effect. The ALIF-SVD dual noise reduction method proposed in this study is compared with the single ALIF, EMD, and EMD-SVD dual noise reduction method through simulation, and the correlation coefficient, signal-to-noise ratio, and mean square error are used to evaluate the noise reduction. It is found that the ALIF-SVD dual noise reduction method avoids the phenomenon of modal aliasing in the decomposition process, effectively removes the noise, and can retain the useful information of the original signal, and the noise reduction effect is better. A unit of a hydropower station in China is further selected as the research object, and its draft tube pressure fluctuation data were analyzed for noise reduction. It was found that this method can accurately extract the signal characteristics under strong noise background, so as to determine the type of pressure fluctuation of the unit, which is helpful to improve the fault recognition rate of hydraulic turbines. And it provides some technical support for the safe and stable operation of hydropower units and the promotion of condition-based maintenance strategy and improves the intelligent level of hydropower station operation management.
\end{abstract}

\section{Introduction}

In recent years, China's hydropower has been fully developed and has produced huge economic benefits, and the stability of hydropower unit operation has attracted more and more attention and research. However, hydropower units are affected by hydraulic factors and other factors in unsteady operation [1-3], and the state quantity related to vibration changes greatly. At the same time, in the process of monitoring the status of each vibration part, due to the influence of the hardware and the environment, the collected vibration signals contain large noise components, which affect the accurate extraction of vibration signal characteristics. Since the diagnosis of hydrogenerator unit faults is mainly based on the frequency of each vibration part, effective noise reduction on the collected data is of great significance to the accurate judgment of unit faults.

At present, there are many vibration signal analysis methods for hydrogenerator units, such as empirical mode decomposition (EMD), local mean decomposition (LMD), 
and ensemble empirical mode decomposition (EEMD). EMD decomposes the signal into a number of IMF components with clear frequency amplitude, which is widely used in the processing of nonlinear and nonstationary signals. However, EMD decomposition is easy to produce modal aliasing, and there are problems such as large amount of iterative calculation [4-7]. In order to overcome the shortcomings of the EMD method, the literature [8] proposed an adaptive signal decomposition method of local mean decomposition, which has a certain improvement in the number of iterations and operation speed compared with EMD, but the problem of modal aliasing was still not fundamentally solved. Aiming at the problems of modal aliasing of EMD, Wu et al. proposed ensemble empirical modal decomposition $[9,10]$, using the uniform distribution of Gaussian white noise frequency and adding Gaussian white noise to the original signal for multiple EMD decomposition, which can effectively suppress modal aliasing. However, it is often difficult to extract early or weak fault signals from mixed signals when using this method alone in practical applications [11-13]. In fact, considering the complexity of the actual signal in actual engineering application, using a hybrid method can extract useful signals from strong interference more effectively than using a single method.

Based on the above analysis, this study proposes a dual noise reduction method based on ALIF-SVD for the fault feature extraction of the pressure pulsation signal of the tailrace pipe of hydropower units under strong noise background, combined with the advantages of adaptive iterative filtering, sample entropy, and singular value. Adaptive local iterative filtering is a decomposition method based on iterative filtering $[14,15]$, and its filtering function is constructed by using the basic solution system of Fokker-Planck (FP) differential equation. The complex multicomponent signal is adaptively decomposed into a number of single-component signals with certain physical meaning by this method. Compared with EMD, this algorithm avoids the problem of data distortion caused by modal aliasing in the decomposition process, thereby ensuring the true validity of the components obtained by decomposition [16-19]. Singular value decomposition (SVD) is carried out on the basis of matrix decomposition and transformation, and the advantages are that the waveform is not easily distorted and the zero-phase shift is small. Compared with EEMD, SVD can effectively detect weak information mutations in the signal under complex background and has outstanding effects in feature information extraction and noise removal [20-22]. Entropy, as a feature extraction method measuring the system's complexity in the time domain, has been maturely applied to fault diagnosis and pathological signal detection [23-25]. Pincus proposed the concept of approximate entropy (AE) based on the theory of Shannon entropy in 1991. However, the $\mathrm{AE}$ has self-matching terms in the calculation, which leads to bias of the result. Thus, in 2000, Richman and Moorman proposed an improvement of the AE, which is the sample entropy (SE). Sample entropy quantifies a system's degree of regularity by calculating the negative natural logarithm of conditional probability, which have high reliability and consistency for data of different lengths. Compared with approximate entropy, sample entropy eliminates the bias caused by self-matching to provide more accurate entropy values, and it also reduces the computational time [26-28].

Through the simulation analysis and example verification, it is found that the method can effectively reduce the noise of the pressure pulsation signal of the tailrace pipe under the background of strong noise and avoid the modal aliasing in the decomposition process. The characteristic frequency of pressure pulsation can be accurately extracted, so as to accurately determine the pulsation type of the unit and provide a theoretical basis for the fault diagnosis of hydrogenerator units.

The content of this study is arranged as follows: In the first part, the principle of the noise reduction method is introduced. In the second part, this method is used for simulation and comparative analysis. In the third part, the pressure fluctuation data of a hydropower station in China are selected for example verification. Finally, the application of this method in the feature extraction of tailpipe pressure pulsation signal under strong noise background is summarized and prospected.

\section{ALIF-SVD Method Principle}

2.1. Principles of Adaptive Iterative Filtering and Sample Entropy. Adaptive local iterative filtering (ALIF) is an improved algorithm based on iterative filtering (IF). The construction of filtering function is based on the basic solution system obtained by Fokker-Planck differential equation, which is adaptive in the decomposition process [14-18]. Therefore, the basic principle of the IF algorithm is introduced first.

2.1.1. Iterative Filtering Algorithm. Each IMF component obtained in the IF algorithm is obtained by iterative screening. This component not only reflects the internal fluctuation characteristics of the original signal to be decomposed but also ensures that a component only contains the characteristic information of one mode. The determination of the IMF component needs to meet the following two conditions at the same time: (1) The difference between the number of extreme points and the number of zero points of the IMF component is not more than 1. (2) At any time point, the average envelope of the upper envelope fitting the maximum value of IMF component and the lower envelope fitting the minimum value of IMF component is equal to 0 , that is, the upper and lower envelopes are locally symmetric relative to the time axis [29-31].

Different from the EMD algorithm, the IF algorithm replaces the cubic spline interpolation in the EMD method to obtain the envelope by constructing a sliding operator. For any given signal to be decomposed $x(t)$ and determine the sliding operator $\Gamma(x(t))$ by calculating the convolution between $x(t)$ and filter function $\omega(t)$, 


$$
\Gamma(x(t))=\int_{-l(z)}^{l(z)} x(t+\tau) \omega(t) \mathrm{d} \tau,
$$

where $\tau$ is the time delay; $t$ is the time; $l(z)$ is the filtering interval; and $\omega(t)$ is the fixed low-pass filter function. The interval of $l(z)$ is determined by the following formula:

$$
l(z)=2 \text { fix }\left(\frac{N \lambda}{m}\right)
$$

where $\lambda$ is the set parameter, and its value range is $1-3 ; m$ is the number of extreme points of the decomposed signal; $N$ is the length of the decomposition signal; and fix is the integral function to 0 .

The specific steps for signal decomposition using IF are as follows:

(1) First, the filtering interval $l(z)$ is calculated by decomposing the signal $x(t)$ and formula (2)

(2) Second, the sliding operator $\Gamma(x(t))$ is calculated by formula (1)

(3) Then, the difference between the decomposed signal and the sliding operator is calculated, and the fluctuation operator is obtained:

$$
k(x(t))=x(t)-\Gamma(x(t))_{1} .
$$

(4) Whether the obtained fluctuation operator $k(x(t))$ satisfies the two conditions for becoming an IMF component is judged. If satisfied, the extraction is completed at one time, that is, $k(x(t))$ is the first extracted IMF component, denoted as $c_{1}(t)$. If not satisfied, $k(x(t))$ is taken as the step for the signal to be decomposed; and repeat (1)-(3) until the IMF component is satisfied.

(5) $x(t)$ minus $c_{1}(t)$

$$
r_{1}(t)=x(t)-c_{1}(t),
$$

Repeat $r_{1}(t)$ as the original signal to (1)-(4) steps to get the second IMF component $c_{2}(t)$ and then repeat $n$ times to get $n$ IMF components satisfying the conditions. When $r_{n}(t)$ shows obvious trend characteristics, the cycle process is terminated. At this time,

$$
x(t)=\sum_{i=1}^{n} c_{i}(t)-r_{n}(t)
$$

After a limited number of screening, $n$ IMF components are obtained.

2.1.2. Adaptive Local Iterative Filtering Algorithm. In the iterative filtering process, since the iterative filtering algorithm is not adaptive, it is necessary to set a low-pass filter function before data processing. Although this method can reduce noise interference to a certain extent, in the process of analyzing nonlinear and nonstationary signals, waveform distortion and poor adaptability will occur [16]. Based on this, the iterative filtering is improved, and the filter function is constructed with the help of the basic solution system obtained from the Fokker-Planck differential equation, which makes it adaptive in the decomposition process.

Let two functions $g(x)$ and $h(x)$ be the derivables on the interval $(a<b<0)$ and satisfy the following conditions:

(1) For any number, there are $g(x)>0$ and $g(a)=g(b)=0$

(2) $h(a)<0<h(b) ; x \in(a, b)$

The formula of Fokker-Planck differential equation is

$$
\frac{\partial p}{\partial t}=-\alpha \frac{\partial(h(x) p)}{\partial x}+\beta \frac{\partial^{2}\left(g^{2}(x) p\right)}{\partial x^{2}} .
$$

where $\alpha$ and $\beta$ are the steady-state coefficients, ranging from 0 to 1 .

In equation (6), $\beta\left(\partial^{2}\left(g^{2}(x) p\right) / \partial x^{2}\right)$ has diffusion, so that the solution $p(x)$ of the equation moves from the middle position of interval $(a, b)$ to both ends. The effect of $-\alpha(\partial(h(x) p) / \partial x)$ is exactly the opposite, which makes the solution $p(x)$ of the equation move from both ends to the middle. When these two items reach a balance, there is

$$
-\alpha \frac{\partial(h(x) p)}{\partial x}+\beta \frac{\partial^{2}\left(g^{2}(x) p\right)}{\partial x^{2}}=0 .
$$

At this time, the differential equation has a nonzero solution and meets the following conditions:

(1) $\forall x \in(a, b), p(x) \geq 0$

(2) $\forall x \notin(a, b), p(x)=0$

The solution $p(x)$ of this differential equation is the required filtering function $\omega(t)$; with the change of interval $(a, b)$, different filtering function expressions will be obtained; so, ALIF realizes the adaptive solution of the filtering function.

2.1.3. Principle of Sample Entropy. Sample entropy (SE) is a method to measure the complexity of time series, which is used to measure the probability of time series generating new patterns when the data dimension changes. The relationship between self-similarity of time series and sample entropy is negatively correlated: when the signal is disturbed by noise, the uncertainty of the signal increases, the disorder and complexity of the signal increases, the sample entropy increases, and the self-similarity of the time series is lower.

The steps to calculate the sample entropy of time series are as follows:

(1) Let $X_{\mathrm{m}}(i)$ be a time series with dimension $m$ and length $N$

$$
\begin{aligned}
X_{m}(i)= & \{x(i), x(i+1), \ldots, x(i+m-1), \\
& \cdot \text { Formula } i=1,2, \ldots, N-m+1
\end{aligned}
$$

(2) Define the distance $\mathrm{d}\left[X_{m}(i), X_{m}(j)\right]$ between vector $X_{\mathrm{m}}(i)$ and vector $X_{\mathrm{m}}(j)$ as the absolute value of the 
maximum difference between the two corresponding data points, namely,

$$
\begin{array}{r}
d\left[X_{\mathrm{m}}(i), X_{m}(j)\right]=\max \left(\left|X_{m}(i+k)-X_{m}(j+k)\right|\right), \\
\text { Formula } k=0,1, \ldots, m-1
\end{array}
$$

(3) Given threshold r, from $1 \sim N-m$ sequences, the number of statistics, $d\left[X_{m}(i), X_{m}(j)\right]<r$, is denoted as $B_{i}$, and the ratio of $B_{i}$ to $N-m-1$ is denoted as $B_{i}^{m}(r)$. Namely,

$$
\begin{aligned}
& B_{i}^{m}(r)=\frac{B_{i}}{N-m-1} \\
& 1 \leq j \leq N-m, j \neq i .
\end{aligned}
$$

The mean value is defined as

$$
\bar{B}_{i}^{m}(r)=\frac{\sum_{i=1}^{N-m} B_{i}^{m}(r)}{N-m} .
$$

(4) For dimension $m+1$, repeat steps (1)-(3) to get $B_{i}^{m+1}(r)$, and further get $\bar{B}^{m+1}(r)$

(5) Theoretically, for a given threshold $r$, the sample entropy of the sequence is defined as

$$
\operatorname{Se}(m, r)=\lim _{N \longrightarrow \infty}\left[-\ln \frac{\bar{B}_{i}^{m+1}(r)}{\bar{B}_{i}^{m}(r)}\right] .
$$

In practical engineering, if $N$ is a finite sequence, it can be estimated that

$$
\operatorname{Se}(m, r, N)=-\ln \frac{\bar{B}_{i}^{m+1}(r)}{\bar{B}_{i}^{m}(r)} .
$$

It can be seen from the above formula that the calculation of sample entropy is related to the values of $m, r$, and $N$. According to the research results of Pincus, when $m=1$ or 2, $r=0.1-0.25$ Std (Std is the standard deviation of the original data $x(i), i=1,2, \ldots, N)$; when $N>500$, the result of sample entropy obtained by calculation is more reasonable in statistical theory $[27,28]$. Therefore, $m=2, r=0.1 \mathrm{Std}$, and $N=6000$ are used in this study.

\subsection{The Principle of Singular Value Difference Spectrum.} Singular value decomposition (SVD) has a wide range of applications in the field of signal analysis. Its denoising is based on the phase space, that is, the correlation matrix is decomposed by singular value decomposition, and the singular value corresponding to the zero noise part is set at the same time. Finally, the nonnoise signal is reconstructed by SVD inverse operation, so as to achieve the effect of noise reduction.

Assuming that there is a signal to be decomposed, $Y=(y(1), y(2), \ldots, y(n))$, and we construct a $m \times n$-order Hankel matrix as follows:

$$
H=\left[\begin{array}{cccc}
y(1) & y(2) & \cdots & y(n) \\
y(2) & y(3) & \cdots & y(n+1) \\
\vdots & \vdots & \vdots & \vdots \\
y(N-n+1) & y(N-n+2) & \cdots & y(N)
\end{array}\right] .
$$

where $N$ is the length of the signal to be decomposed, $1<n<N ; m=N-n+1 ; H \in R^{m * n}$ 。.

SVD decomposition of the obtained matrix, the operation is as follows:

$$
\begin{gathered}
H=\mathrm{USV}^{T}, \\
\text { Formula } S=\left\{\begin{array}{l}
\left(\operatorname{diag}\left(\sigma_{1}, \sigma_{2}, \ldots, \sigma_{q}\right), 0\right) ; m \leq n, \\
\left(\operatorname{diag}\left(\sigma_{1}, \sigma_{2}, \ldots, \sigma_{q}\right), 0\right)^{T} ; m>n .
\end{array}\right.
\end{gathered}
$$

$U=\left(u_{1}, u_{2}, \ldots, u_{m}\right) \in R^{m * n}$ $V=\left(v_{1}, v_{2}, \ldots, v_{n}\right) \in R^{m * n}$ are the two orthogonal matrices; $S \in R^{m * n}$ is determined by the relationship between $m$ and $n$; 0 represents zero matrix; $q=\min (m, n)$; and then, the singular value of matrix $H$ is $\sigma_{1} \geq \sigma_{2} \geq \cdots \geq \sigma_{q} \geq 0$.

When a signal is created by a Hankel matrix, the number of rows $m$ and columns $n$ of the matrix can be determined according to the following principles $[19,20]$; when the signal length $N$ is even, take $m=(N+1) / 2, n=(N / 2)$, and at this moment, take the maximum $q=(N / 2)$. When the signal length $N$ is odd, take $m=(N+1) / 2, n=(N+1) / 2$, and take maximum $q=(N+1) / 2$. The Hankel matrix constructed by this method is decomposed and reconstructed by SVD, and the noise reduction effect is good.

It is necessary to select the useful singular value order reasonably for signal reconstruction, and the singular value difference spectrum well expresses the singular value mutation of noisy signal.

The sequence formed by singular values arranged in the descending order is set to $\delta_{i}=(i=0,1,2, \ldots, q)$; then, the former singular values are subtracted from the latter singular values, that is, $b_{i}=\delta_{i}-\delta_{i+1}=0,1,2, \ldots, q-1$; then, the new sequence composed of $b_{i}$ is a singular value difference spectrum. According to the definition of difference spectrum, since the correlation between the useful signal and the noise is different, the two signals show the maximum difference in the singular value, forming the peak value in the difference spectrum. Therefore, the maximum mutation point is the boundary point of the two signals. If the maximum mutation occurs at the position of point $S$, when the reconstruction order is carried out, the noise reduction effect can be achieved by selecting the one before the $S$ point [20-22].

\subsection{ALIF-SVD Signal Noise Reduction Method Steps. (1)}

First, ALIF decomposition is performed on the collected signal to be decomposed according to formulas (1)-(7) to obtain a series of IMF components

(2) The sample entropy of the IMF component is calculated according to formulas (10)-(13) 
(3) According to the results of multiple simulation experiments and the principle of sample entropy calculation, the sample entropy threshold is set as 0.7, and select some IMF components for reconstruction

(4) Perform SVD decomposition on the reconstructed signal according to (15), and select the appropriate singular value to reconstruct again according to the decomposed singular value difference spectrum

(5) Analyze the characteristic frequency of the reconstructed signal to judge the unit failure

And the technical route is shown in Figure 1:

\section{Simulation Signal and Analysis}

The draft tube of a hydraulic turbine generally has lowfrequency, intermediate-frequency, and high-frequency pressure pulsations. In order to simulate the pressure pulsation of the turbine draft tube, the simulation signal is constructed. Assuming that the unit rotation frequency is $2 \mathrm{~Hz}$, according to the characteristics of the actual hydropower unit draft tube pulsation frequency, the simulation signal is set to four characteristic frequencies of $0.5 \mathrm{~Hz}$, $1.1 \mathrm{~Hz}, 2 \mathrm{~Hz}$, and $9.5 \mathrm{~Hz}$, and a random Gaussian white noise is considered. The sampling frequency is set to $500 \mathrm{~Hz}$, and the simulation waveform formula is set as

$$
\begin{aligned}
x 1= & 0.35 \sin (1 \pi t)+0.3 \sin (2.2 \pi t) \\
& +0.2 \sin (4 \pi t)+0.2 \sin (19 \pi t), \\
x 2= & 1.5 \text { randn }(1: 5000) \\
x 3= & x 1+x 2 .
\end{aligned}
$$

where $\mathrm{x} 1$ is the noise-free signal, $\mathrm{x} 2$ is the noise signal, $\mathrm{x} 3$ is the noise-added signal, and $t$ is the time.

The simulation waveform and spectrum without noise and the waveform and spectrum after noise are shown in Figures 2 and 3. It can be seen from the figure that the simulated signal waveform after adding noise is abnormally messy, the characteristic frequency is disturbed by the noise frequency, and the fault characteristic is difficult to extract in the spectrum diagram, which will seriously affect the accurate judgment of the unit fault.

In order to accurately extract the characteristics of weak vibration signals under strong noise background, the double noise reduction method of ALIF-SVD proposed in this study is used to process the simulation signal. At the same time, in order to verify the superiority of this method, this method is compared with a single ALIF, EMD, and EMD-SVD dual noise reduction method, and the results are shown in Figures 4-7.

It can be seen from Figures 4-7 that the frequencies of $0.5 \mathrm{~Hz}, 1.1 \mathrm{~Hz}, 2 \mathrm{~Hz}$, and $9.5 \mathrm{~Hz}$ set by the simulation are extracted after four methods of processing. However, the signal after ALIF, EMD, and EMD-SVD noise reduction processing has more interference frequencies, the bottom noise and amplitude are larger, the vibration waveform of the data cannot be fully and clearly displayed, and the waveform processed by EMD and EMD-SVD is obviously

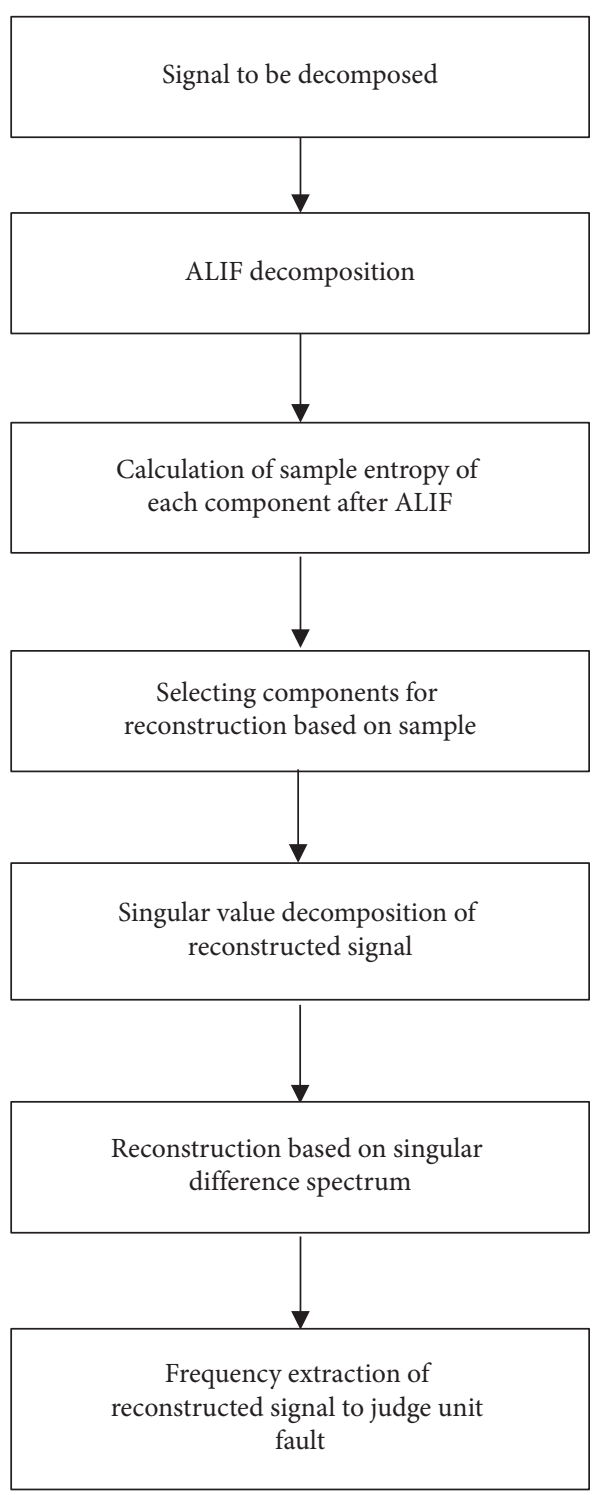

FIGURE 1: Denoising flowchart.

distorted. After ALIF-SVD denoising processing, the waveform and graph 2 (simulation without noise waveform and spectrum diagram) are very close, the reduction degree is very high, the signal integrity is good, and there is no distortion and leakage of data. It shows that the dual noise reduction method of ALIF-SVD can effectively remove noise, accurately extract the frequency characteristics of vibration signals, ensure data integrity, and achieve relatively better noise reduction effects.

In order to more scientifically reflect the advantages and disadvantages of the noise reduction effects of the above four methods, the correlation coefficient, signal-to-noise ratio, and mean square error are calculated, respectively, as judgment indicators, and the noise reduction effects of the four methods are quantitatively analyzed.

The correlation between the original signal and the denoised signal is expressed by the correlation coefficient. The larger the correlation coefficient, the better the correlation, and the smaller the signal distortion after denoising 


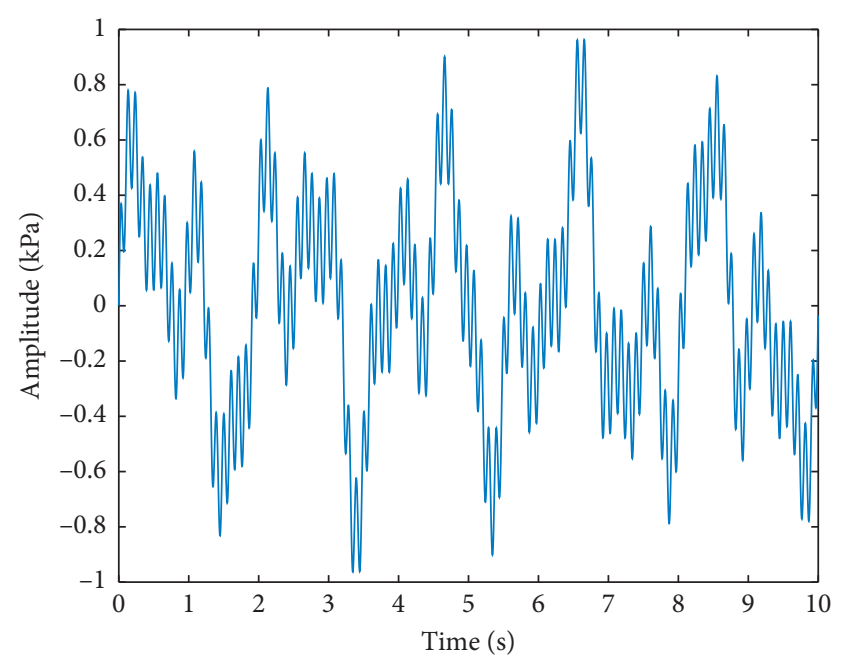

(a)

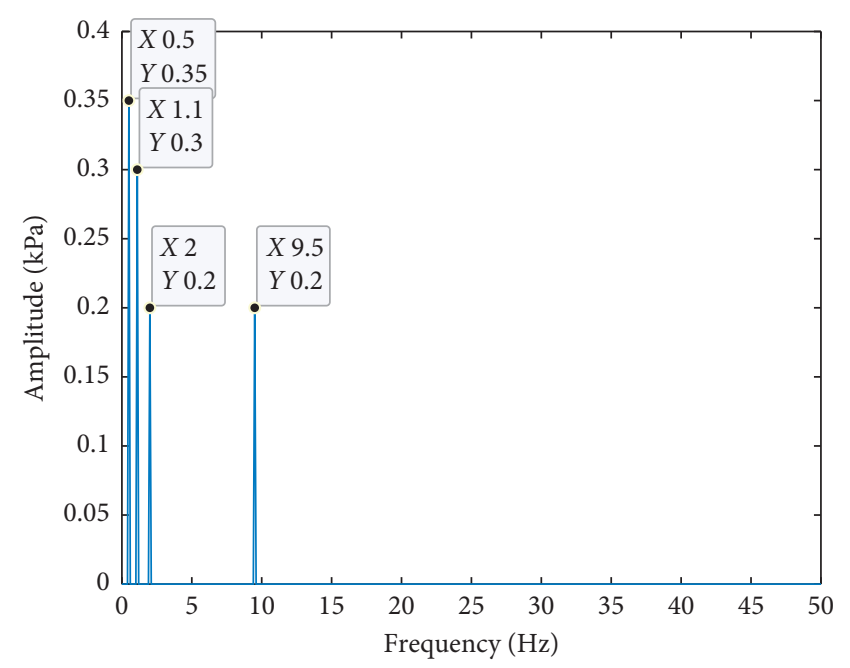

(b)

FigURE 2: Simulation without noise waveform and spectrum diagram. (a) Waveform diagram. (b) Spectrogram.

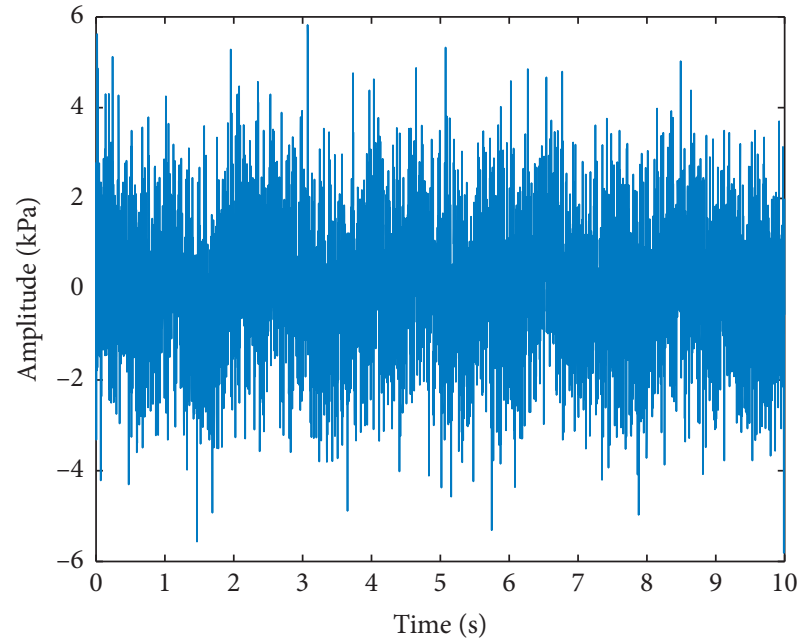

(a)

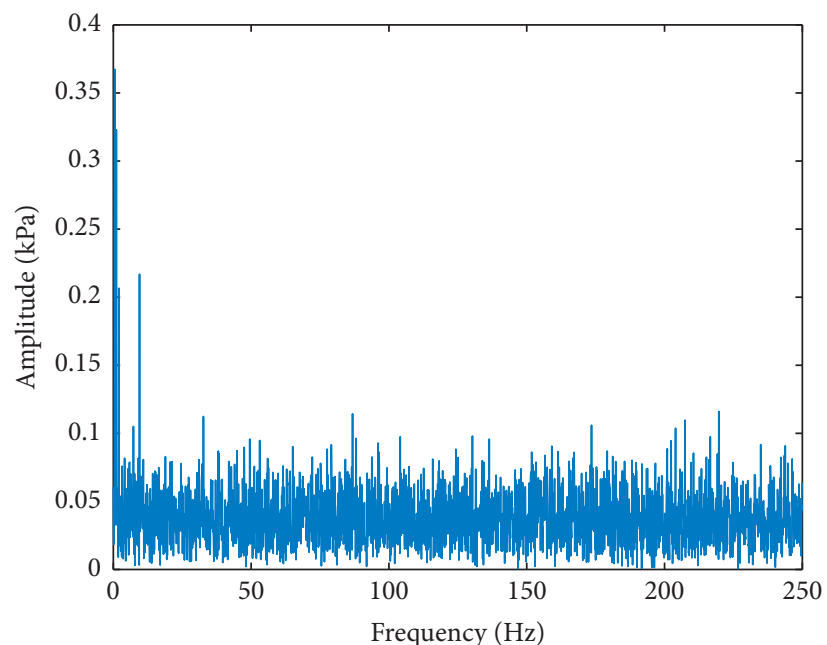

(b)

FiguRE 3: Simulation of noise addition waveform and spectrum diagram. (a) Waveform diagram. (b) Spectrogram.

is, the more useful information the original signal retains. The range of value is $0-1$ [32-34]. The formula is as follows:

$$
r(a(t), b(t))=\frac{\operatorname{cov}(a(t), b(t))}{\sqrt{D(a(t)) D(b(t))}},
$$

where $a(t)$ is the simulation signal without noise data, and $b(t)$ is the data obtained after denoising.

The ratio of signal energy to noise energy is expressed by the signal-to-noise ratio, which is positively correlated with the denoising effect, that is, the larger the value, the better the denoising effect [32-34]. The formula is as follows:

$$
\mathrm{SNR}=10 \lg \frac{\sum c(t)^{2}}{\sum[c(t)-b(t)]^{2}},
$$

where $c(t)$ is the original signal data.
The square root of the difference between the denoised signal and the original signal is represented by the mean square error. This value represents the degree of signal distortion after denoising, and the smaller the value is, the better it is [32-34]. The formula is as follows:

$$
\text { RMSE }=\sqrt{\frac{1}{n} \cdot \sum_{i=1}^{n}[f(i)-f \prime(i)]^{2}},
$$

where $f(i)$ is the original signal; $f_{\prime}(i)$ is the signal after denoising; and $n$ is the signal length.

Calculate the relevant judgment indicators as given in Table 1. It can be seen that after ALIF-SVD denoising, the correlation of data is as high as 0.9700 , the signal-to-noise ratio is larger, and the mean square error is smaller. It further shows that the method can ensure the integrity of the signal, 


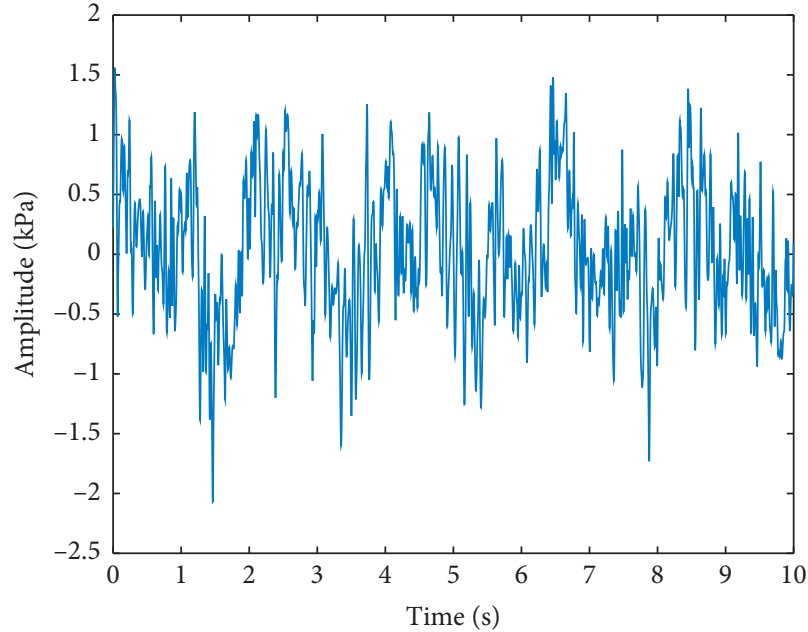

(a)

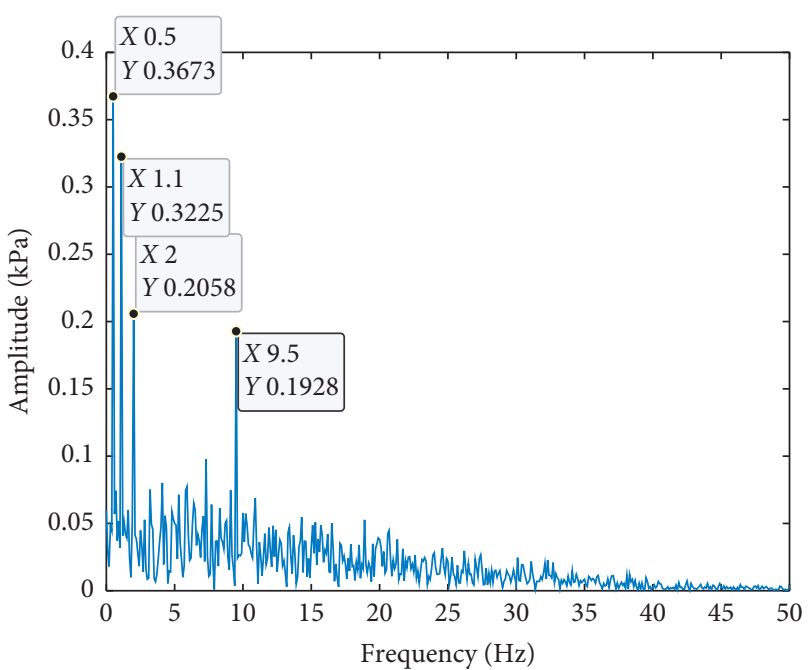

(b)

FIgURE 4: Waveform and spectrum diagram after ALIF noise reduction. (a) Waveform diagram. (b) Spectrogram.

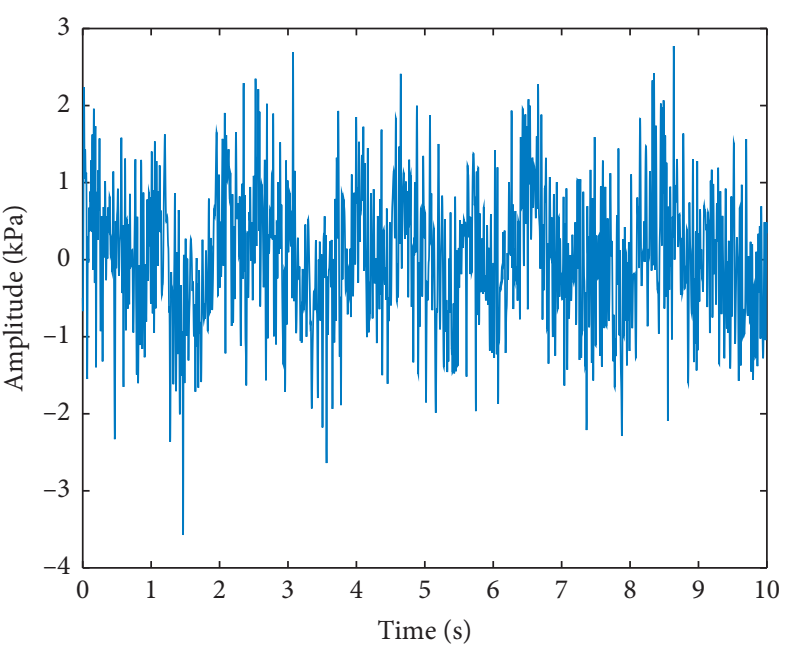

(a)

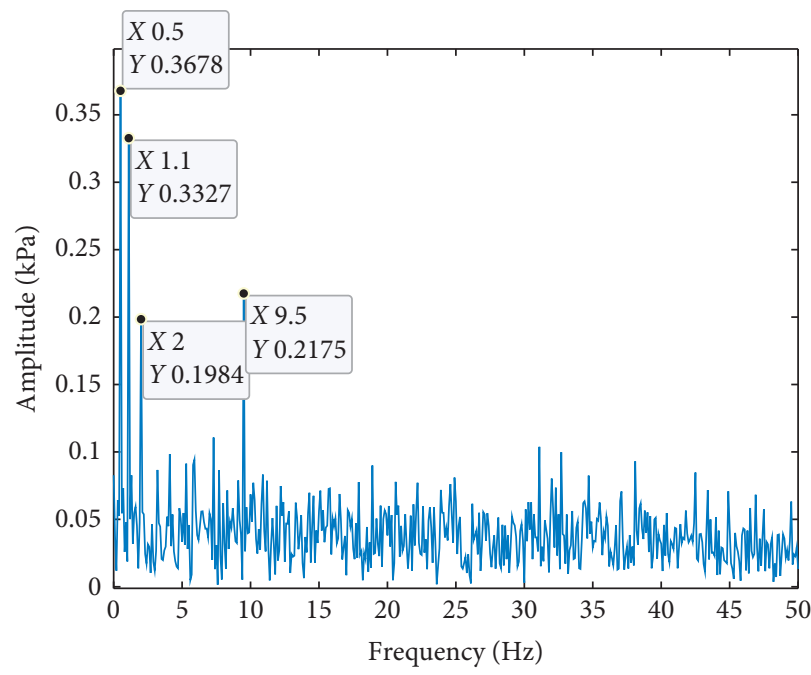

(b)

FIGURE 5: Waveform and spectrum diagram after EMD noise reduction. (a) Waveform diagram. (b) Spectrogram.

retain more useful information, and have a better denoising effect. It is very suitable for extracting weak signal characteristics under strong noise background.

\section{Example Verification}

It can be seen from the simulation signal experiment that the single ALIF and EMD denoising methods have a great gap compared with the dual denoising method of ALIF-SVD proposed in this study, but compared with the dual denoising method of EMD-SVD, the denoising effect of this method is better. In order to better illustrate the effectiveness of this method, the actual measurement signal is verified by this method.

The pressure fluctuation data of the draft tube of a certain hydropower station in China are selected for analysis. The turbine model is HL702-LJ-410, the unit speed is $136 \mathrm{r} / \mathrm{min}$, and the data acquisition frequency is $500 \mathrm{~Hz}$. When the unit output is $63 \mathrm{MW}$, the data are collected for $2 \mathrm{~min}$, and 5000 data points are selected for analysis, and use of the dual ALIF-SVD noise reduction methods is processed.

The waveform and spectrum of the tailpipe data and the waveform and spectrum of the tailpipe after ALIF-SVD denoising are shown in Figures 8 and 9.

It can be seen from the figure that the actual spectrum of the draft tube is full of noise, and the pulsation frequency is covered by the noise frequency, so it is difficult to extract the weak vibration noise. After the ALIF-SVD denoising method proposed in this study, the fluctuating waveform of the draft tube can be clearly displayed, the frequency is clear, and there is almost no noise interference. 


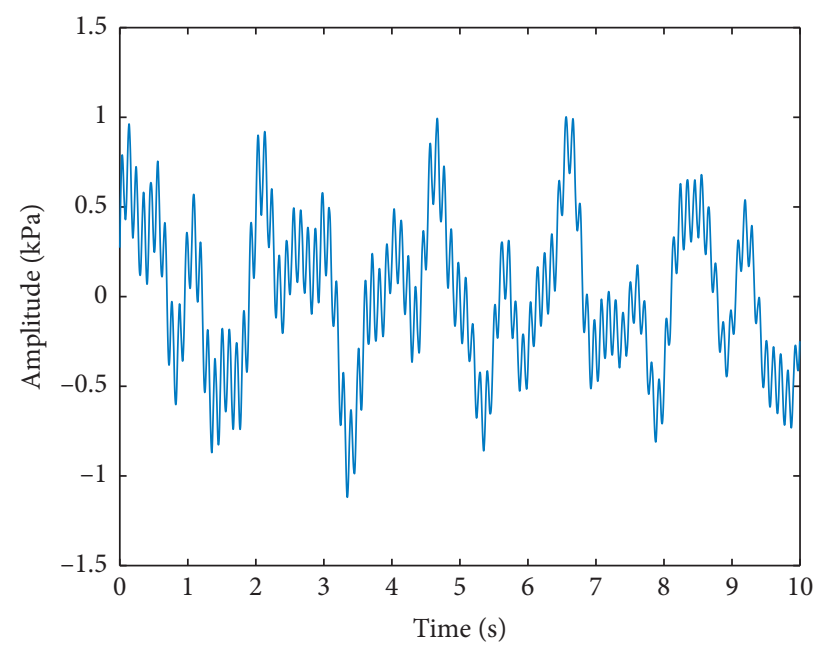

(a)

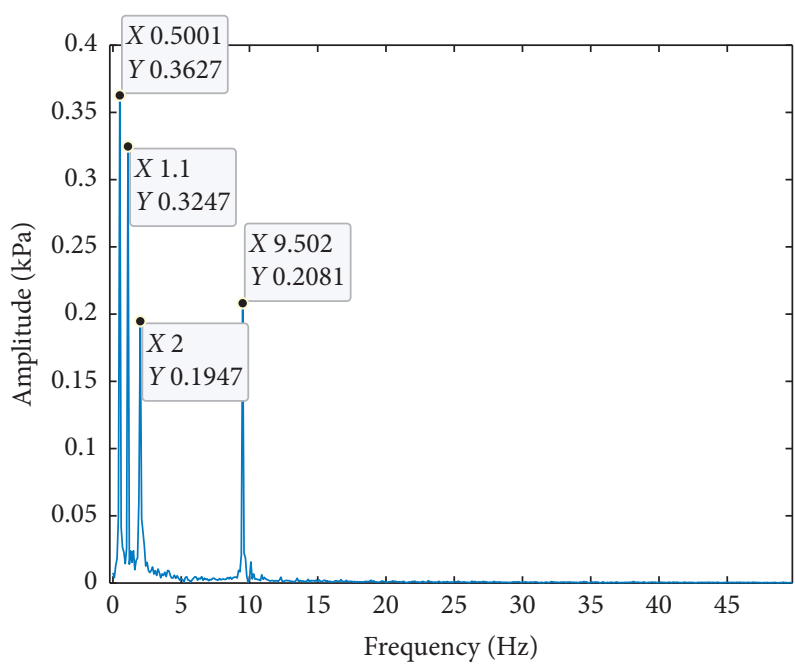

(b)

FIGURE 6: Waveform and spectrum diagram after EMD-SVD noise reduction. (a) Waveform diagram. (b) Spectrogram.

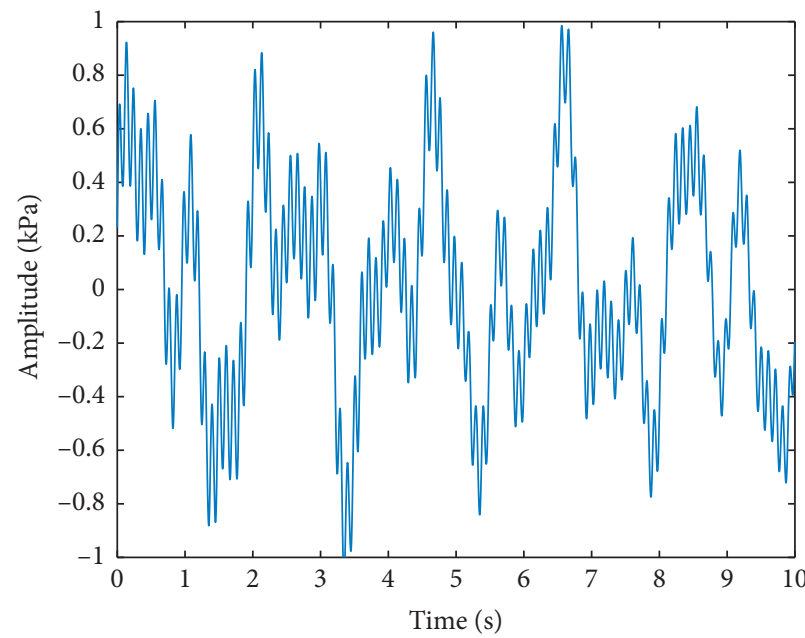

(a)

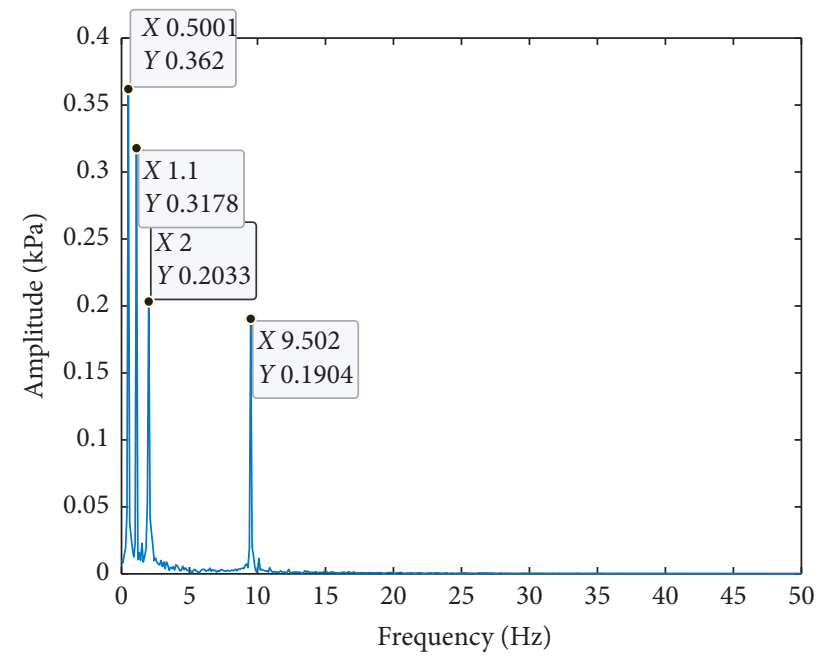

(b)

FIgURE 7: Waveform and spectrum diagram after ALIF-SVD noise reduction. (a) Waveform diagram. (b) Spectrogram.

TABLE 1: Related index values.

\begin{tabular}{lccc}
\hline & Correlation coefficient & Signal-to-noise ratio & Mean square error \\
\hline ALIF & 0.7091 & 3.0043 & 0.3961 \\
EMD & 0.5016 & 1.2394 & 0.6984 \\
EMD-SVD & 0.9591 & 10.9018 & 0.1175 \\
ALIF-SVD & 0.9700 & 12.2365 & 0.0987 \\
\hline
\end{tabular}

There are four frequencies of $0.2 \mathrm{~Hz}, 0.6 \mathrm{~Hz}, 1.1 \mathrm{~Hz}$, and $11 \mathrm{~Hz}$ in the spectrum diagram. According to the literature [35], $0.2 \mathrm{~Hz}, 0.6 \mathrm{~Hz}$, and $1.1 \mathrm{~Hz}$ are the low-pressure pulsation frequencies of the draft tube, and $11 \mathrm{~Hz}$ is the high- pressure pulsation frequency of the draft tube. Therefore, it can be judged that there are low-pressure pulsation and high-pressure pulsation in the tail water pipe of the unit, and the low-pressure pulsation is the main one. 


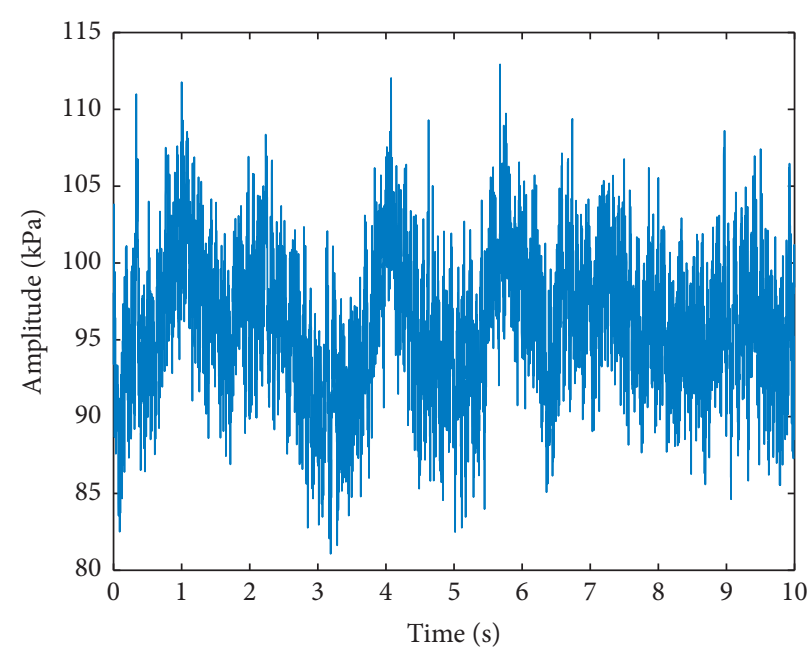

(a)

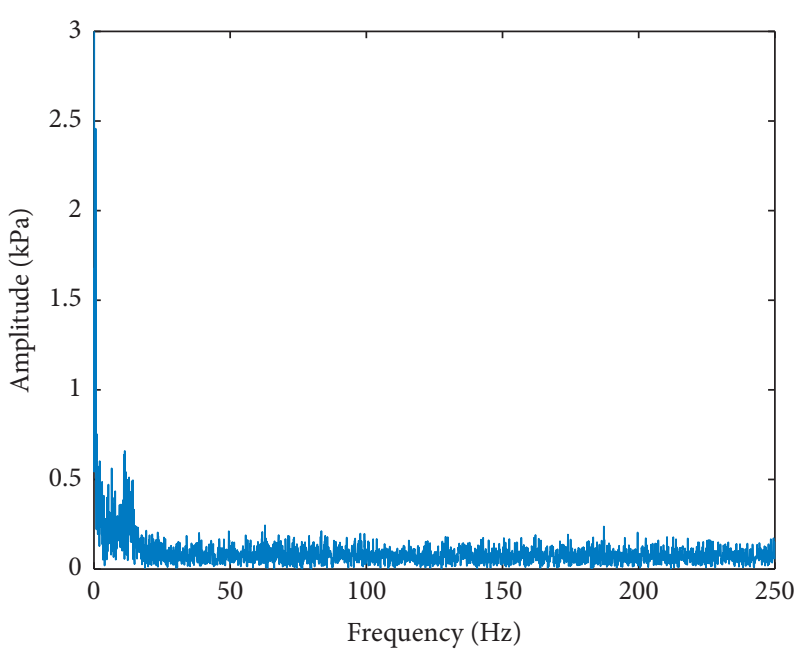

(b)

FigURE 8: Waveform and spectrum diagram of the draft tube. (a) Waveform diagram. (b) Spectrogram.

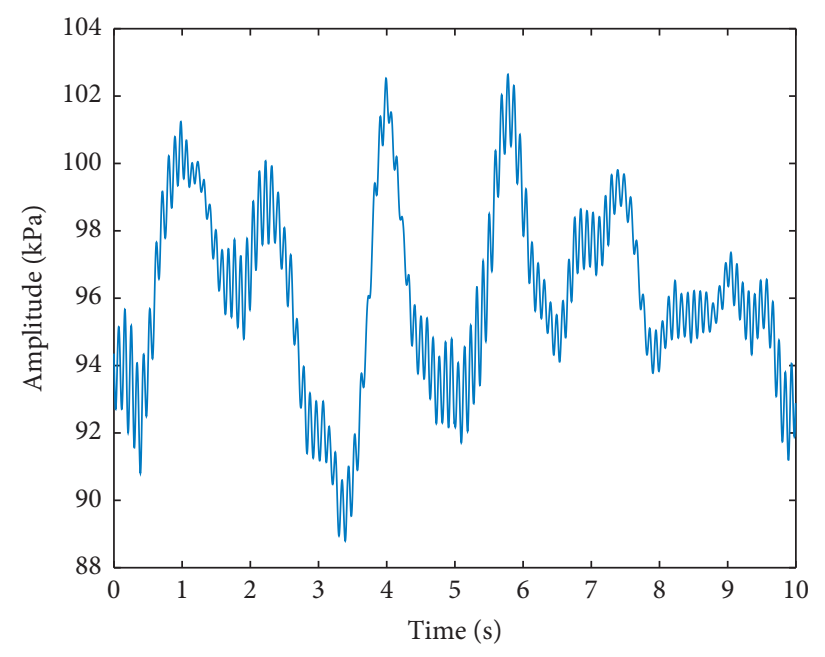

(a)

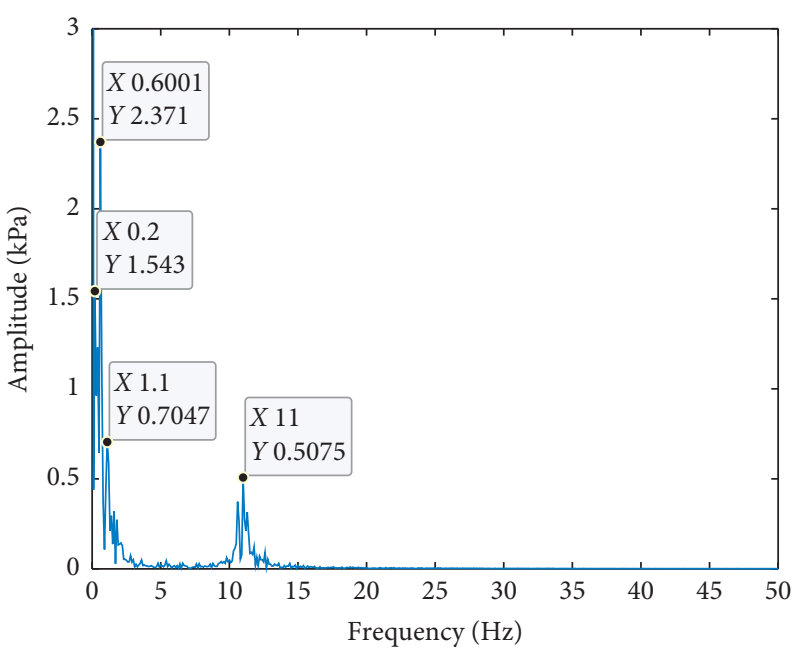

(b)

FIGURE 9: Waveform and spectrum diagram of the draft tube after ALIF-SVD noise reduction. (a) Waveform diagram. (b) Spectrogram.

\section{Conclusion}

In this study, by combining the advantages of adaptive local iterative filtering, sample entropy, and singular value decomposition, a dual denoising method based on ALIF-SVD is proposed to solve the problem that weak feature signals are difficult to extract under strong noise background.

(1) Through the simulation experiment, the ALIF-SVD noise reduction method is compared with the single ALIF, EMD, and the EMD-SVD double noise reduction method, and the correlation coefficient, signal-to-noise ratio, and mean square error are calculated at the same time. It is found that after signal denoising by this method, the mode mixing phenomenon in the EMD decomposition process can be avoided, and the data with correlation coefficient as high as 0.97 , higher signal-to-noise ratio, and smaller mean square error can be obtained. The noise can be effectively removed, and the useful information of the original signal can be retained to achieve a good denoising effect.

(2) Through the experimental analysis of the measured tailrace pipe pulsation data of the hydrogenerator unit, it is found that this method can double denoising the pulsating signal of the draft tube, effectively remove the noise component, and accurately extract the characteristic frequency of the pulsation signal. Thus, the pulsation type of the unit can be judged by the frequency, which can greatly reduce the diagnosis time of the power station for complex hydraulic faults in the actual operation. 


\section{Data Availability}

The data used to support the findings of this study are available from the corresponding author upon request.

\section{Conflicts of Interest}

The authors declare that they have no conflicts of interest.

\section{Acknowledgments}

The study was supported by the following fund projects: (1) Henan Province Key R \& D and Promotion Project (Science and Technology Research) in 2021 (212102311054); (2) Training Program for Young Key Teachers in Colleges and Universities of Henan Province in 2019 (2019GGJS097); (3) Key Scientific Research Projects of Colleges and Universities in Henan Province in 2017 (17A480006); (4) Hunan Key Laboratory of Renewable Energy Power Technology (Changsha University of Technology) Open Fund Project (2016ZNDL001); Project of Excellent Online Open Courses in Colleges and Universities of Henan Province in 2020 《Hydraulic turbine》(Item number: 92).

\section{References}

[1] L. Shi, W. Zhang, H. Jiao et al., "Numerical simulation and experimental study on the comparison of the hydraulic characteristics of an axial-flow pump and a full tubular pump," Renewable Energy, vol. 153, pp. 1455-1464, 2020.

[2] L. Shi, J. Zhu, F. Tang et al., "Multi-disciplinary optimization design of axial-flow pump impellers based on the approximation model," Energies, vol. 13, 779 pages, 2020.

[3] C. Luo, D. Zhang, C. Zhang et al., "Investigation of rotating stall phenomenon and optimization in mixed-flow waterjet propulsion device," Advances in Materials Science and Engineering, vol. 2020, no. 3, 17 pages, Article ID 9421809, 2020.

[4] S. Kunju, S. Shi, C. Jiang, and H. Zhang, "Rolling bearing feature frequency extraction using extreme average envelope decomposition," Chinese Journal of Mechanical Engineering, vol. 79, 2015.

[5] Yaguo, J. Lei, Z. He, and M. J. Zuo, "A review on empirical mode decomposition in fault diagnosis of rotating machinery," Mechanical Systems and Signal Processing, vol. 35, pp. 108-126, 2013.

[6] D. Wu, Z. Shengjie, Z. Huimin et al., "A novel fault diagnosis method based on integrating empirical wavelet transform and fuzzy entropy for motor bearing," IEEE Access, p. 1, 2018.

[7] M. Van, H. J. Kang, and K. S. Shin, "Rolling element bearing fault diagnosis based on non-local means de-noising and empirical mode decomposition," IET Science, Measurement and Technology, vol. 8, no. 6, pp. 571-578, 2014.

[8] C. Jun-Sheng, Y. Yu, and Y. U. De-Jie, "The local mean decomposition method and its application to gear fault diagnosis," Journal of Vibration Engineering, vol. 22, no. 1, pp. 76-84, 2009.

[9] W. Tong, M. Zhang, Q. Yu et al., "Comparing the applications of EMD and EEMD on time-frequency analysis of seismic signal," Journal of Applied Geophysics, vol. 83, no. 29-34, pp. 29-34, 2012.

[10] T. Y. Wu and Y. L. Chung, "Misalignment diagnosis of rotating machinery through vibration analysis via the hybrid
EEMD and EMD approach," Smart Materials and Structures, vol. 18, no. 9, Article ID 095004, 2009.

[11] Y. Lei and M. J. Zuo, "Fault diagnosis of rotating machinery using an improved HHT based on EEMD and sensitive IMFs," Measurement Science and Technology, vol. 20, no. 12, p. 125701, 2009.

[12] X. T. Wu, M. Yang, X. H. Yuan et al., "Bearing fault diagnosis using EEMD and improved morphological filtering method based on kurtosis criterion," Zhendong Yu Chongji/Journal of Vibration and Shock, vol. 34, no. 2, pp. 38-44, 2015.

[13] H. Jiang, C. Li, and H. Li, "An improved EEMD with multiwavelet packet for rotating machinery multi-fault diagnosis," Mechanical Systems and Signal Processing, vol. 36, no. 2, pp. 225-239, 2013.

[14] A. Cicone, J. Liu, and H. Zhou, "Adaptive local iterative filtering for signal decomposition and instantaneous frequency analysis," Applied and Computational Harmonic Analysis, vol. 41, no. 2, pp. 384-411, 2016.

[15] M. Piersanti, M. Materassi, A. Cicone et al., "Adaptive local iterative filtering: a promising technique for the analysis of nonstationary signals," Journal of Geophysical Research: Space Physics, 2018.

[16] X. An, H. Zeng, and C. Li, "Demodulation analysis based on adaptive local iterative filtering for bearing fault diagnosis," Measurement, vol. 94, pp. 554-560, 2016.

[17] Y. Lv, Y. Zhang, and C. Yi, "Optimized adaptive local iterative filtering algorithm based on permutation entropy for rolling bearing fault diagnosis," Entropy, vol. 20, no. 12, 2018.

[18] L. Zhao, Y. Zhang, and D. Zhu, "Rolling element bearing fault diagnosis based on adaptive local iterative filtering decomposition and teager-kaiser energy operator," Journal of Failure Analysis and Prevention, no. 4, 2019.

[19] X. An, H. Zeng, W. Yang et al., "Fault diagnosis of a wind turbine rolling bearing using adaptive local iterative filtering and singular value decomposition," Transactions of the Institute of Measurement \& Control, Article ID 0142331216644041, 2016.

[20] H. Jiang, J. Chen, G. Dong, T. Liu, and G. Chen, "Study on Hankel matrix-based SVD and its application in rolling element bearing fault diagnosis," Mechanical Systems and Signal Processing, vol. 52-53, pp. 338-359, 2015.

[21] R. Golafshan and K. Yuce Sanliturk, "SVD and Hankel matrix based de-noising approach for ball bearing fault detection and its assessment using artificial faults," Mechanical Systems and Signal Processing, vol. 70-71, no. MAR., pp. 36-50, 2016.

[22] J. Xu, S. Tong, F. Cong, and J. Chen, "Slip Hankel matrix series-based singular value decomposition and its application for fault feature extraction," IET Science, Measurement \& Technology, vol. 11, no. 4, pp. 464-472, 2017.

[23] A. . Humeau-Heurtier, "Evaluation of systems' irregularity and complexity: sample entropy, its derivatives, and their applications across scales and disciplines," Entropy, vol. 20, no. 10, 2018.

[24] A. Howedi, A. Lotfi, and A. Pourabdollah, "Exploring entropy measurements to identify multi-occupancy in activities of daily living," Entropy, vol. 21, no. 4, p. 416, 2019.

[25] C. Chang, S. Shixue, C. Zhixin et al., "A comprehensive comparison and overview of $\mathrm{R}$ packages for calculating sample entropy," Biology Methods and Protocols no. 1, , p. 1.

[26] Y. Li, X. Chen, J. Yu et al., "The data-driven optimization method and its application in feature extraction of ship-radiated noise with sample entropy," Energies, vol. 12, 2019.

[27] W. Li, X. Shen, and Y. Li, "A comparative study of multiscale sample entropy and hierarchical entropy and its application in 
feature extraction for ship-radiated noise," Entropy, vol. 21, no. 8, p. 793, 2019.

[28] R. K. Udhayakumar, C. Karmakar, and M. Palaniswami, "Understanding irregularity characteristics of short-term HRV signals using sample entropy profile," IEEE Transactions on Biomedical Engineering, vol. 65, no. 11, pp. 2569-2579, 2018.

[29] B. Xuan, Q. Xie, and S. Peng, "EMD sifting based on bandwidth," IEEE Signal Processing Letters, vol. 14, no. 8, pp. 537-540, 2007.

[30] N. E. Huang, Z. Shen, S. R. Long et al., "The empirical mode decomposition and the Hilbert spectrum for nonlinear and non-stationary time series analysis," in Proceedings of the Royal Society of London. Series A: Mathematical, Physical and Engineering Sciences, vol. 454, no. 1971, pp. 903-995, 1998.

[31] C. Junsheng, Y. Dejie, and Y. Yu, "Research on the intrinsic mode function (IMF) criterion in EMD method," Mechanical Systems and Signal Processing, vol. 20, no. 4, pp. 817-824, 2006.

[32] T. Ke, "A hybrid indicator for determining the best decomposition scale of wavelet denoising," Acta Geodaetica Et Cartographica Sinica, vol. 41, no. 5, pp. 749-755, 2012.

[33] Y. Chen, L. J. Chen, H. L. Liu et al., "Determination of the optimal decomposition scale in wavelet de-noising of optical fiber sensing signal," Guangdianzi Jiguang/Journal of Optoelectronics Laser, vol. 24, no. 12, pp. 2372-2376, 2013.

[34] L. Lv, W. Gong, S. Song et al., "De-noising process based on wavelet transform in feature reflectance detection LiDAR system," Geomatics and Information Science of Wuhan University, vol. 36, no. 1, pp. 56-59, 2011.

[35] F. Zhang, Z. X. Gao, L. P. Pan et al., "Study on pressure fluctuation in Francis turbine draft tubes during partial load," Journal of Hydraulic Engineering, vol. 42, no. 10, pp. 12341238, 2011. 